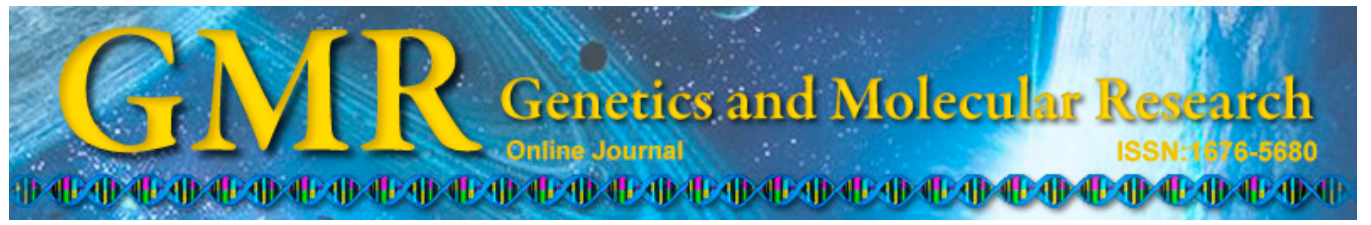

\title{
High-throughput sequencing identification of genes involved with Varroa destructor resistance in the eastern honeybee, Apis cerana
}

\author{
T. Ji, L. Yin, Z. Liu, F. Shen and J. Shen \\ College of Animal Science and Technology, Yangzhou University, \\ Yangzhou, China
}

Corresponding author: T. Ji

E-mail: tji@yzu.edu.cn

Genet. Mol. Res. 13 (4): 9086-9096 (2014)

Received January 23, 2014

Accepted May 15, 2014

Published October 31, 2014

DOI http://dx.doi.org/10.4238/2014.October.31.24

\begin{abstract}
Varroa destructor is the greatest threat to the honeybee Apis mellifera worldwide, while it rarely causes serious harm to its native host, the Eastern honeybee Apis cerana. The genetic mechanisms underlying the resistance of A. cerana to Varroa remain unclear. Thus, understanding the molecular mechanism of resistance to Varroa may provide useful insights for reducing this disease in other organisms. In this study, the transcriptomes of two A. cerana colonies were sequenced using the Illumina Solexa sequencing method. One colony was highly affected by mites, whereas the other colony displayed strong resistance to $V$. destructor. We determined differences in gene expression in the two colonies after challenging the colonies with $V$. destructor. After de novo transcriptome assembly, we obtained 91,172 unigenes for A. cerana and found that 288 differentially expressed genes varied by more than 15 -fold. A total of 277 unigenes were present at higher levels in the non-affected colony. Genes involved in resistance to Varroa included unigenes related to skeletal muscle movement, olfactory sensitivity, and transcription factors. This suggests that hygienic behavior and grooming behavior
\end{abstract}


may play important roles in the resistance to Varroa.

Key words: Apis cerana; Gene expression; Transcriptome; Varroa destructor

\section{INTRODUCTION}

Varroa destructor is a parasitic mite that both physically and physiologically damages honeybees, Apis mellifera, by feeding on the hemolymph of developing and adult bees (Rosenkranz et al., 2009). V. destructor is also a vector and inducer of several highly pathogenic honeybee viruses that severely damage bee colonies, often leading to colony death (Annoscia et al., 2012). V. destructor spread from its native host Apis cerana to A. mellifera four decades ago and later spread to nearly every part of the world. This organism has become the greatest threat to A. mellifera health worldwide (Cornman et al., 2012). A. mellifera is vulnerable to Varroa; however, the mites rarely cause serious damage to their native host $A$. cerana. In some regions of China, it is difficult to identify Varroa mites in A. cerana populations. Natural selection of benign host-parasite interactions may have promoted the co-existence between $A$. cerana and Varroa (Warrit and Lekprayoon, 2011). Several factors have been implicated, including grooming, hygienic behavior, and differences in developmental timing. The genetic mechanisms underlying the resistance to Varroa mites remain unclear. Resistant and susceptible lines of $A$. mellifera have been used to study gene expression and identify resistant genes using microarrays (Navajas et al., 2008; Parker et al., 2012) and to identify chromosomal regions containing genes that affect specific traits using quantitative trait locus (QTL) mapping (Guzman-Novoa et al., 2011; Behrens et al., 2012; Arechavaleta-Velasco and Guzmán-Novoa, 2001; Tsuruda et al., 2012). However, it is difficult to compare these studies because they used different breeding lines of bees with different genetic backgrounds. Thus, the candidate genes and QTLs could not be confirmed in other studies. Furthermore, the level of resistance to Varroa mites is likely regulated by several genes and markers, and A. cerana may have the proper combination of characteristics to confer resistance. Rath and Drescher (1990) suggested that it is necessary to clarify the relationship between Varroa and A. cerana to understand the relationship between Varroa and A. mellifera. Thus, understanding the molecular mechanism of Varroa resistance in A. cerana may explain the lack of resistance of $A$. mellifera to Varroa.

Previous studies reported that $V$. destructor was present in some A. cerana colonies in the Guangdong Province, where it caused visible damage to the colonies (Zhou, 2005). We obtained these bees and designated them as $\mathrm{M}$ bees (bees susceptible to mites). We compared the head-specific gene expression profiles of these bees with those of common A. cerana (C), which shows resistance to $V$. destructor. The Illumina Solexa sequencing method was used to analyze the transcriptome of $A$. cerana workers and we identified differences in gene expression between A. cerana $\mathrm{M}$ and $\mathrm{C}$ workers after challenge by Varroa. The objective of this study was to identify genes that may be involved in resistance to $V$. destructor parasitism, which may provide insights into the mechanism of $A$. mellifera resistance.

\section{MATERIAL AND METHODS}

\section{A. cerana colonies and Varroa mite challenge}

We obtained $A$. cerana from colonies in which $V$. destructor was present. We desig- 
nated these bees as $\mathrm{M}$ bees. We compared the head-specific gene expression profiles of $\mathrm{M}$ bees with those of $A$. cerana $(\mathrm{C})$ that displayed good resistance to $V$. destructor. The $\mathrm{M}$ and $\mathrm{C} A$. cerana colonies were unrelated local strains in the same $A$. cerana population and were bred in the Guangdong Entomological Institute, Guangzhou, China.

We selected 2 combs from the infested brood of $A$. mellifera colonies containing mature female mites in worker brood cells. The 2 combs contained roughly equal numbers of capped worker brood cells and capped drone brood cells. The 2 combs were introduced into $A$. cerana colonies $\mathrm{C}$ and $\mathrm{M}$. After being challenged for $24 \mathrm{~h}$, colonies $\mathrm{C}$ and $\mathrm{M}$ were evaluated for infections and nurse workers were collected from the two A. cerana colonies.

\section{Sibling estimation by microsatellite genotyping}

To minimize the effects of intra-colonial genetic variation on gene expression, equal pools of full-sister workers (related by $75 \%$ because of haplodiploidy) were collected from the two colonies (Navajas et al., 2008). To identify full sisters, 100 bees from each colony were genotyped based on their Ap53 and A107 microsatellite loci (Franck et al., 1999).

\section{Total RNA extraction, cDNA preparation, and sequencing}

Whole heads of workers were freeze-dried in nitrogen at $-70^{\circ} \mathrm{C}$. The RNA extraction procedure used TRIzol ${ }^{\circledR}$ Reagent (Invitrogen, Carlsbad, CA, USA) according to the manufacturer protocol for animal tissues.

Beads coupled to oligo(dT) were used to isolate poly(A) mRNA. The mRNA was disrupted to produce short fragments. First-strand cDNA was synthesized using a random hexamer-primer, while the second-strand cDNA was synthesized using RNaseH and DNA polymerase I (Takara, Shiga, Japan). After end preparation and poly-A tailing, the short fragments were connected with sequencing adapters. The products were purified and enriched by polymerase chain reaction (PCR) to create the final cDNA library. Finally, the library was sequenced using an Illumina HiSeq ${ }^{\mathrm{TM}} 2000$ (Illumina, Inc., San Diego, CA, USA).

\section{De novo transcriptome assembly}

De novo transcriptome assembly was carried out using the short read assembly program Trinity (Grabherr et al., 2011). Trinity was used to combine reads of a certain length, overlapping them to form longer fragments (contigs), and the reads were mapped back to contigs. Next, Trinity was used to connect the contigs and identify sequences that could not be extended at either end (unigenes). Finally, the unigenes produced by $\mathrm{M}$ and $\mathrm{C}$ bee assemblies were processed by sequence splicing and redundancy removal using sequence clustering software to acquire the maximum number of non-redundant unigenes. In the final step, BLASTX alignments (E-value $<0.00001)$ were constructed using the unigenes and protein databases, including GenBank nr, Swiss-Prot, KEGG, and COG. The best alignment results were used to determine the sequence directions of unigenes. If the results obtained using the different databases conflicted, the priority order of GenBank nr, Swiss-Prot, KEGG, and COG was followed to determine the sequence direction of unigenes. If a unigene was not aligned using these databases, ESTScan was used to determine its sequence direction. 


\section{Identification of differentially expressed genes (DEGs)}

Unigene expression was calculated using the reads per kb per million reads (RPKM) method (Mortazavi et al., 2008). Differences in the expression between 2 samples were calculated using the false discovery rate (FDR) method. FDR was used to determine the P value thresholds in multiple tests and analyses. In our analysis, we used FDR $\leq 0.001$ and a ratio $>$ 2 to identify significant DEGs. The DEGs were used in GO functional analysis and KEGG pathway analysis.

\section{DEGs quantification by reverse transcription-quantitative (q)PCR}

High-throughput sequencing results were confirmed by measuring the expression of 9 differentially regulated genes using real-time qPCR.

\section{RESULTS}

\section{Resistance to $V$. destructor}

After challenge by $V$. destructor for $24 \mathrm{~h}$, the $\mathrm{C}$ and $\mathrm{M}$ colonies were evaluated for infections. Colony $\mathrm{C}$ had opened and removed most of the infested worker and drone brood cells of A. mellifera. Chewed mites were observed in the debris of colony $\mathrm{C}$ and no mite-infested A. cerana pupae were found. In contrast, colony $\mathrm{M}$ had opened few cells and a small number of mites were observed in the debris, while some mite-infested drone pupae were observed.

\section{Assembly and functional annotation of the transcriptome}

Two pools of mRNA samples from the $\mathrm{M}$ and $\mathrm{C}$ colonies were used to build libraries that were subjected to high-throughput parallel sequencing using Illumina sequencing. We generated 91,172 all-unigenes from both samples with an average length of 1858 base pairs (bp), a final all-unigene N50 length of $3774 \mathrm{bp}$ (50\% of assembled bases were incorporated into all-unigenes measuring $\geq 3774 \mathrm{bp}$ ), and a total length of $169,402,078 \mathrm{bp}$. Genomic information for A. cerana was lacking, and hence, we performed BLAST alignments (E-value $<1.0$ x $10^{-5}$ ) against the GenBank nr, NT, SwissProt, GO, and KEGG databases to identify putative functions for the all-unigene sequences. A total of 63,496 all-unigenes $(69.6 \%$ of all) matched with 1 or more of the databases, while more than half of the unigenes $(55.04 \%)$ matched to $A$. mellifera in the GenBank nr database, and $26.57 \%$ matched Apis florea (Table 1).

Table 1. Species distribution of the BLASTX results after comparison with the GenBank nr database.

\begin{tabular}{lcr}
\hline Species & Gene number & Percentage \\
\hline Apis mellifera & 28486 & $55.04 \%$ \\
Apis florea & 13752 & $26.57 \%$ \\
Bombus impatiens & 2362 & $4.56 \%$ \\
Bombus terrestris & 2190 & $4.23 \%$ \\
Megachile rotundata & 1049 & $2.03 \%$ \\
Harpegnathos saltator & 480 & $0.93 \%$ \\
Other & 3438 & $6.64 \%$ \\
\hline
\end{tabular}


Based on the GO classifications, 85,577 unigenes were categorized into biological processes $(85,577$ members), cellular components $(48,194$ members), and molecular functions $(29,301$ members) (Figure 1). We also searched the annotated sequences for genes with COG classifications. A total of 23,790 sequences had COG classifications (Figure 2). We also mapped the annotated sequences to reference canonical pathways in the KEGG database. We assigned a total of 39,625 sequences to 258 KEGG pathways (able S1).

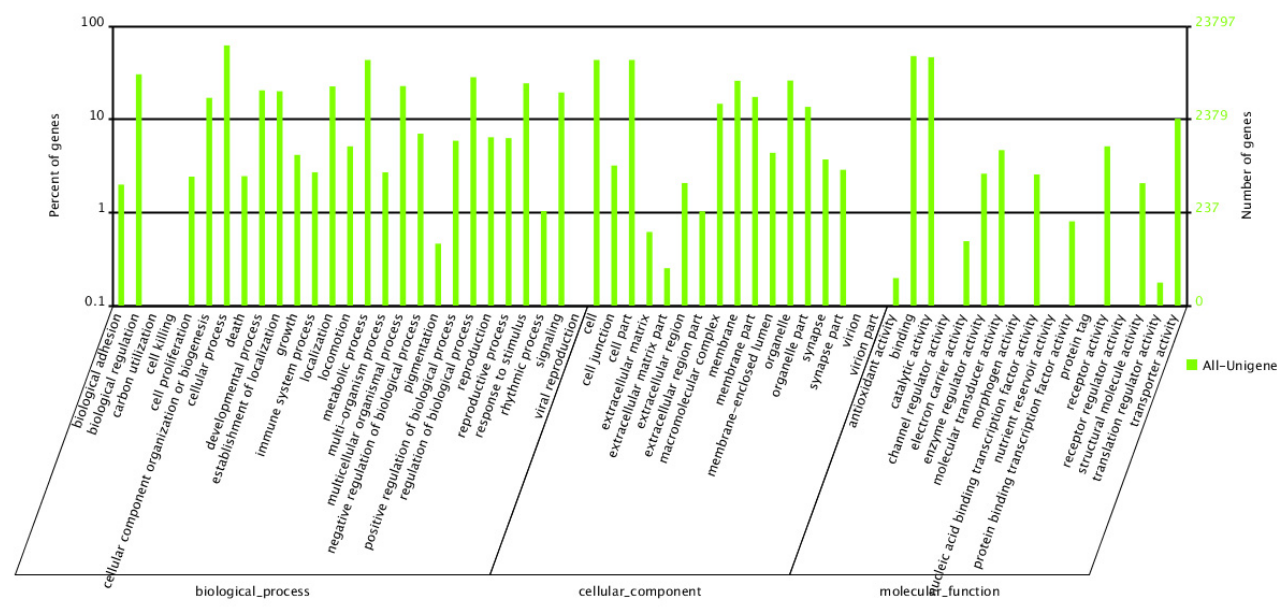

Figure 1. Histogram presentation of Gene Ontology classification. The results are summarized in 3 main categories: biological process, cellular component, and molecular function. The right $y$-axis indicates the number of genes in a category. The left y-axis indicates the percentage of a specific category of genes in that main category.

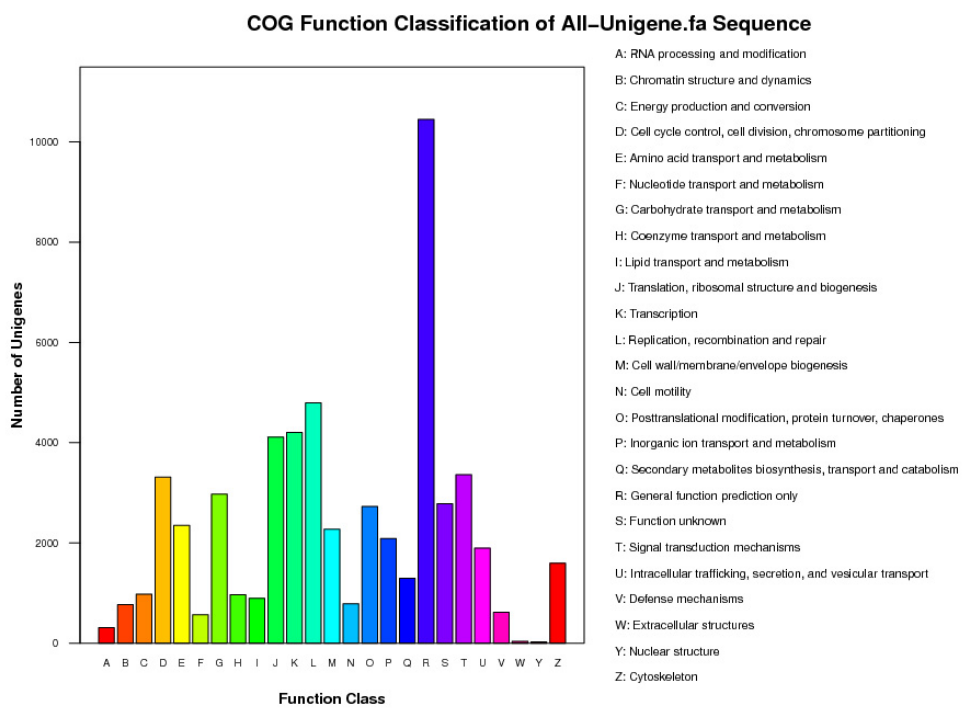

Figure 2. Histogram presentation of clusters of orthologous groups (COG) classification. The histogram shows the distribution of sequences among different COG categories: 23,790 sequences have a COG classification among the 25 categories. 


\section{DEGs in colonies $\mathrm{M}$ and $\mathrm{C}$}

Differences in the gene expression levels in the $\mathrm{M}$ and $\mathrm{C}$ libraries were estimated and used to scan for genes associated with resistance to Varroa. FDR $<0.001$ and an absolute value of $\mid \log _{2}$ ratio $\mid \geq 1$ were used as thresholds to determine the statistical significance of gene expression. Transcripts with at least a 2 -fold difference in the 2 libraries are shown in Figure 3. We identified 40,255 genes that were differently expressed in C and M. Of these, 20,553 were upregulated and 19,702 were downregulated in colony C.

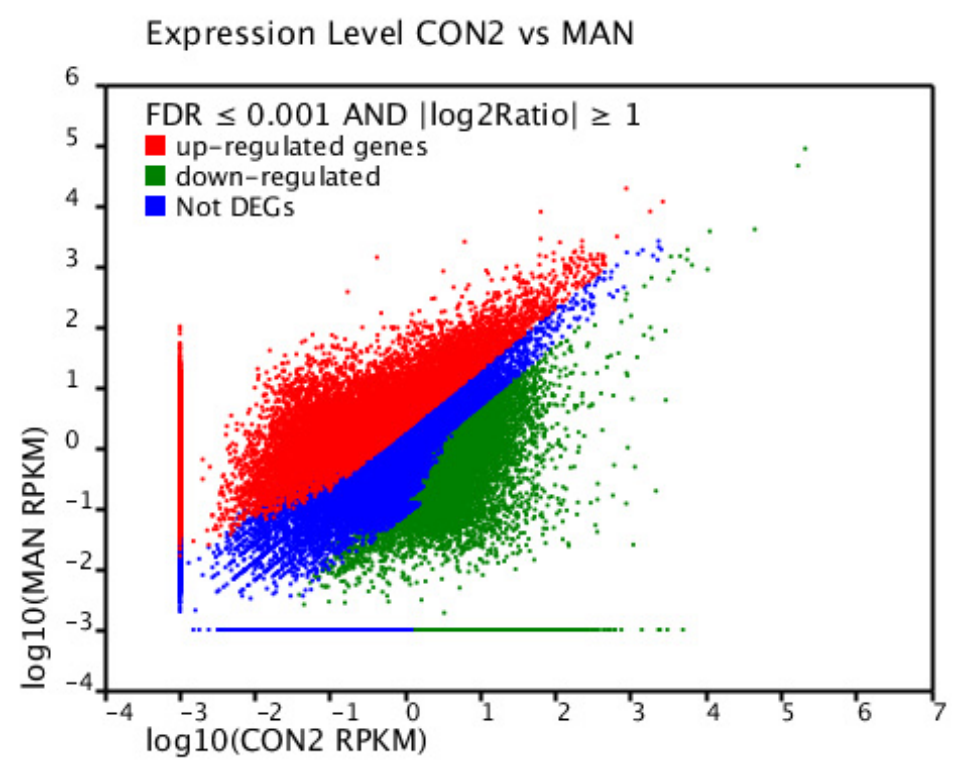

Figure 3. Comparison of gene expression levels in the colony M (MAN) and C (CON2) libraries. To compare gene expression levels, each library was normalized using 1,000,000 tags. Red dots indicate transcripts that were more prevalent in the $\mathrm{M}$ library, green dots indicate transcripts that were more prevalent in the $\mathrm{C}$ library, and blue dots indicate transcripts that were similar in both libraries. FDR $\leq 0.001$ and $\mid \log _{2}$ ratio $\mid \geq 1$ were used as thresholds to determine the significance of gene expression differences.

\section{High expression transcripts in colonies $\mathrm{M}$ and $\mathrm{C}$}

We found that the DEGs showed greater than 15 -fold differences and that 277 unigenes were present at higher levels in colony $\mathrm{C}$ than colony $\mathrm{M}$, whereas 11 were present at lower levels (Table S2), suggesting that many more genes were highly upregulated in colony $\mathrm{C}$ than in colony $\mathrm{M}$ when parasitized by Varroa mites. Of the upregulated unigenes in colony M, 11 DEGs were associated with skeletal muscle, including troponin (5 unigenes), calcium-transporting ATPase (1 unigene), and sarcalumenin (2 unigenes). Three members of the odorant binding protein (OBP) family (obp4, obp17, and obp18) were significantly upregulated in colony $\mathrm{C}$. Two transcription factors were significantly upregulated in colony $\mathrm{C}$, including cAMP-responsive element-binding protein (CREB)-like 2-like and transcription factor mblk-1-like (Mblk-1). We randomly selected 9 genes and designed 18 pairs of primers (Table 2) for qPCR validation. Expression patterns were consistent with the observed 
findings in transcriptome analysis (Figure 4). Their significant agreement indicated that the abundance of the Illumina sequences from the $A$. cerana transcriptome closely mirrored the actual expression level.

\section{Table 2. Primers for relative real-time quantitative PCR (qPCR).}

\begin{tabular}{ll}
\hline Primer name & Sequence \\
\hline Troponin C type IIb & GGCTGCACACTCTCTTCCTC \\
Odorant binding protein 17 & TCCCAATCCGAATGAATGAT \\
& TGTTGAGCAGTGCCAATTTC \\
Odorant receptor Or2-like & TGCTATTTGCGTTTGCGTTA \\
Troponin H $(T p n H)$ and troponin I $(T p n I)$ genes & ATACGGCAGCATCAATCGTT \\
Ankyrin & TCTCGTACGTGTCGAAGCAC \\
Aromatic-L-amino-acid decarboxylase-like & GACATCGACCAATCGCATTA \\
Transcription factor mblk-1-like (Mblk-1) & GCCTAACGAGTCTCCTGCAC \\
Twitchin-like & GACAAGGTTAAAGCCGTTGC \\
Tropomyosin-1-like & TCGTCCGGTATCGTTTTCTC \\
& TCAACCAGTGCGTTTGAAAG \\
& TGCTGCAACTGGGACAATAA \\
& GGGGAATCTTAAAGGCGAAG \\
& TGCCAAGCACTACACCAAAA \\
& GTCGTCACTGTGTGGTCGTC \\
& CGGTATTTCGTGCGGTTAGT \\
& ACTAAAGGAGGCCGAAGCTC \\
\hline
\end{tabular}

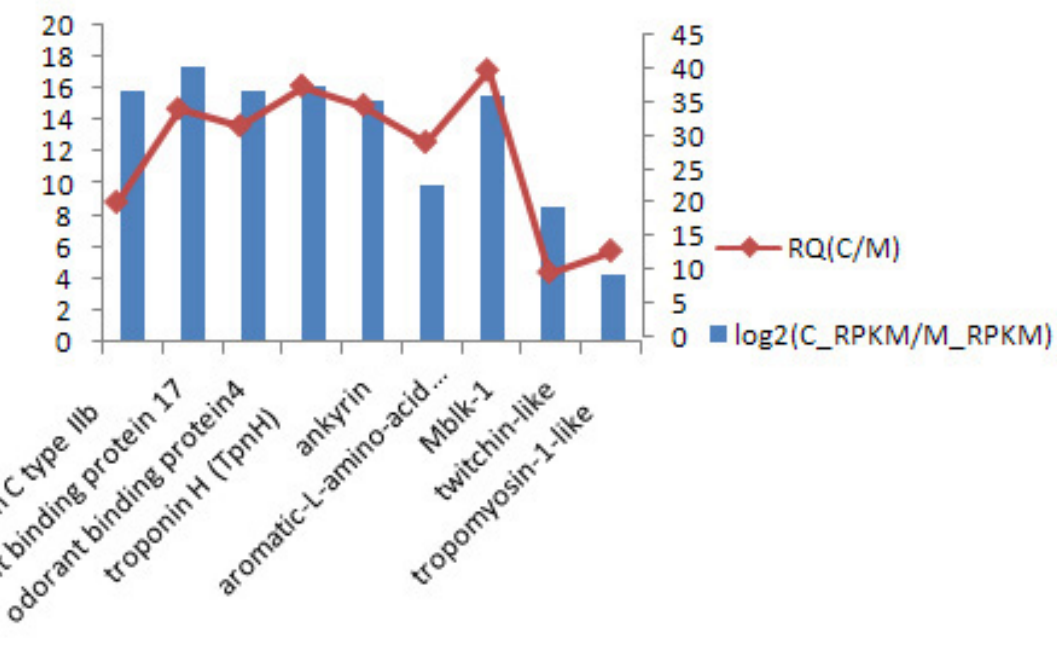

Figure 4. Validation of candidate genes in Apis cerana transcriptome using qPCR. The right $y$-axis indicates the relative abundance of candidate genes resulting from qPCR. The left y-axis indicates gene expression levels of candidate genes according to RPKM calculations.

\section{DISCUSSION}

Illumina RNA-Seq technology is an efficient method for identifying novel genes and investigating gene expression patterns, particularly in organisms in which the genome has not 
been sequenced (Yao et al., 2012). We used high-throughput sequencing data to determine differential gene expression in two A. cerana colonies challenged by $V$. destructor. Colony M was susceptible to mites, whereas colony $\mathrm{C}$ showed good resistance to $V$. destructor. Several genes were identified that may be involved in $V$. destructor resistance.

Troponin (Tn) is involved in striated muscle excitation and contraction and it is the main regulatory protein in these processes. The Tn complex is composed of 3 subunits, i.e., troponin C (TnC), troponin I (TnI), and troponin $\mathrm{T}(\mathrm{TnT})$, which mediate the thin-filament response to calcium when striated muscular contraction is initiated. In mammalian systems, structure-function relationships between the Tn complex subunits have been examined previously (O'Connell et al., 2008). Insect studies have focused on the function of troponin in the development of the muscles responsible for flight in the adult thorax. High expression of $\mathrm{Tn}$ in the heads of bees from colony $\mathrm{C}$ indicated that striated muscles in their heads engaged in strong contraction and relaxation when infected with Varroa mites. In skeletal muscle, the calcium-transporting ATPase sarcoplasmic/endoplasmic reticulum type can control muscular contraction and heat production; this activity can signal the efficient metabolism of various cell types (Toyoshima and Nomura, 2002; Sørensen et al., 2004). Sarcalumenin is a $\mathrm{Ca}^{2+}$-binding protein located in the sarcoplasmic reticulum of striated muscle cells. Its physiological function has not been fully determined, but some studies have shown that sarcalumenin affected store-operated $\mathrm{Ca}^{2+}$ entry and enhanced muscle fatigue resistance in sarcalumenin knockout mice (Zhao et al., 2005; Yoshida et al., 2005). The large difference in the expression of calcium-transporting ATPase and sarcalumenin in colonies $\mathrm{C}$ and $\mathrm{M}$ also suggested that colony $\mathrm{C}$ engaged in stronger muscular movements when infected with Varroa mites. Several studies have shown that grooming and hygienic behaviors are the most important mechanisms for A. cerana resistance to Varroa. Grooming behavior has been studied in A. mellifera and was shown to occur at a lower frequency than that in A. cerana (Bienefeld et al., 1999). There have also been reported that mites were injured by worker bees using their mandibles (Arechavaleta-Velasco and Guzmán-Novoa, 2001). Worker bees of $A$. cerana (nurse-aged worker bees) can also detect capped broods infested with $V$. destructor; the bees open infested cells to remove the mites. This behavior is known as hygienic or removal behavior (Boecking, 1992). Both of these behaviors involve the use of muscles in the head, particularly in the mandibles, so the high expression of troponin, calcium-transporting ATPase, and sarcalumenin indicate that these genes have important roles in grooming and hygienic behaviors.

Many studies have suggested that bees with the highest olfactory sensitivity generally initiate hygienic behavior because they can detect and accurately discriminate between abnormal and normal broods at a low stimulus intensity (Martin et al., 2001; Swanson et al., 2009). Differences in olfaction may account for bee tolerance of Varroa and may also be related to increased grooming and hygienic behavior, which are important behaviors known to be involved with Varroa tolerance (Navajas et al., 2008). The recognition and discrimination of thousands of odorous compounds is mediated by olfactory sensory neurons. In insects, chemosensory neurons are surrounded by an aqueous milieu that acts as a barrier to volatile and primarily lipophilic molecules. Thus, many airborne molecules, such as hydrophobic odorants and pheromones, must be recognized by a specialized class of proteins that facilitate their delivery to the olfactory receptors. It is now widely accepted that this function is provided by OBPs in insects (Deyu and Leal, 2002). Thus, upregulation of 3 OBPs 
may be related to the greater olfactory sensitivity of colony C. Of the three odorant-binding proteins, only obp4 was expressed exclusively in the antennae of adult bees in A. mellifera, whereas obp17 and obp18 were expressed ubiquitously in all adult body parts (Forêt and Maleszka, 2006). This suggests that obp17 and obp18 are involved in other physiological functions. Thus, obp 4 may be the most important gene for sensitive olfactory detection by the $\mathrm{C}$ colony. Furthermore, the Down syndrome cell adhesion molecule gene, which is also involved in olfaction, was upregulated in colony $\mathrm{C}$, although the difference in expression level was not as high (13.7496). Differential expression of this group of genes in different bee genotypes is very important given that olfaction may play an important role in detecting Varroa-infested cells in colony C.

Two transcription factors were significantly upregulated in colony $\mathrm{C}$, including CREB-like 2-like and transcription factor Mblk-1. Mblk-1 is a transcription factor that may function in the mushroom body (MB) neuronal circuits and it is preferentially expressed in the MB in the honeybee brain (Takeuchi et al., 2001; Park et al., 2003). Grooming behavior and hygienic behavior are social behaviors in honeybees that may require complex processing of sensory information in the brain. However, little is known regarding the molecular basis of their highly advanced behaviors. MBs are thought to be involved in sensory integration, learning, and memory in insects (Groh et al., 2006). Honeybee MBs are well-developed compared with those in other insects (Blenau and Erber, 1998; McQuillan et al., 2012), suggesting that MB function is closely associated with advanced honeybee behaviors. Because it is preferentially expressed in the MB, Mblk-1 and its target genes may have important roles in grooming behavior and hygienic behavior.

CREB is a cAMP-responsive transcription factor that has been implicated in the activation of the synthesis of proteins required for long-term facilitation in a cellular model of memory (Warburton et al., 2005). The cAMP signaling system has critical roles in the production of long-term memory (LTM) and long-term synaptic plasticity that may underlie LTM formation in insects. Activation of CREB leads to the protein synthesis-dependent long-term synaptic plasticity underlying LTM formation (Belvin and Yin, 1997; Chen et al., 2012). This suggests that LTMs were formed when $A$. cerana initiated the resistance reaction to Varroa mites, such as olfactory learning (Decourtye et al., 2005; Dupuis et al., 2012). Thus, A. cerana may generate an LTM of specific odors of $V$. destructor so that the workers can recognize the mites more easily during the next infestation. Therefore, colony $\mathrm{C}$ may have a better ability to store LTMs of specific odors than colony M.

Behavior is affected by heritable and environmental factors, and occasionally by massive changes in the brain transcriptome (Robinson, 2004). Behavior-specific neurogenomic states can be inferred based on the coordinated actions of transcription factors and their predicted target genes (Chandrasekaran et al., 2011). Identification of possible target genes for Mblk-1 and CREB and their biological functions may provide important clues regarding the molecular basis of the mechanism of Varroa resistance in A. cerana.

\section{ACKNOWLEDGMENTS}

Research supported by the Earmarked Fund for Modern Agro-industry Technology Research System from the Ministry of Agriculture of China (\#CARS-45-SYZ6) and the National Natural Science Foundation of China (\#31372382) and the Graduate Education Innovation Project in Jiangsu Province, China (\#CXZZ13-0911). 


\section{Supplementary material}

\section{REFERENCES}

Annoscia D, Del Piccolo F and Nazzi F (2012). How does the mite Varroa destructor kill the honeybee Apis mellifera? Alteration of cuticular hydrcarbons and water loss in infested honeybees. J. Insect Physiol. 58: 1548-1555.

Arechavaleta-Velasco ME and Guzmán-Novoa, E (2001). Relative effect of four characteristics that restrain the population growth of the mite Varroa destructor in honey bee (Apis mellifera) colonies. Apidologie 32: 157-174.

Behrens D, Huang Q, Gebner C, Rosenkranz P, et al. (2012). Three QTL in the honey bee Apis mellifera L. suppress reproduction of the parasitic mite Varroa destructor. Ecol. Evol. 1: 451-458.

Belvin MP and Yin JC (1997). Drosophila learning and memory: recent progress and new approaches. Bioessays 19: 1083-1089.

Bienefeld K, Zautke F, Pronin D and Mazedd A (1999). Recording the proportion of damaged Varroa jacobsoni in the debris of honey bee colonies (Apis mellifera). Apidologie 30: 249-256.

Blenau W and Erber J (1998). Behavioural pharmacology of dopamine, serotonin and putative aminergic ligands in the mushroom bodies of the honeybee (Apis mellifera). Behav. Brain Res. 96: 115-124.

Boecking (1992). Removal behavior of Apis mellifera towards sealed brood cells infested with Varroa jacobsoni: techniques, extent and efficacity. Apidologie 23: 371-373.

Chandrasekaran S, Ament SA, Eddy JA, Rodriguez-Zas SL, et al. (2011). Behavior-specific changes in transcriptional modules lead to distinct and predictable neurogenomic states. Proc. Natl. Acad. Sci. U. S. A. 108: 18020-18025.

Chen CC, Wu JK, Lin HW, Pai TP, et al. (2012). Visualizing long-term memory formation in two neurons of the Drosophila brain. Science 335: 678-685.

Cornman RS, Tarpy DR, Chen Y, Jeffreys L, et al. (2012). Pathogen webs in collapsing honey bee colonies. PLoS One 7: e43562.

Decourtye A, Devillers J, Genecque E, Le Menach K, et al. (2005). Comparative sublethal toxicity of nine pesticides on olfactory learning performances of the honeybee Apis mellifera. Arch. Environ. Contam. Toxicol. 48: 242-250.

Deyu Z and Leal W (2002). Conformational isomers of insect odorant-binding proteins. Arch. Biochem. Biophys. 397 : 99-105.

Dupuis J, Louis T, Gauthier M and Raymond V (2012). Insights from honeybee (Apis mellifera) and fly (Drosophila melanogaster) nicotinic acetylcholine receptors: from genes to behavioral functions. Neurosci. Biobehav. Rev. 36: 1553-1564.

Forêt S and Maleszka R (2006). Function and evolution of a gene family encoding odorant binding-like proteins in a social insect, the honey bee (Apis mellifera). Genome Res. 16: 1404-1413.

Franck P, Coussy H, Le Conte Y, Solignac M, et al. (1999). Microsatellite analysis of sperm admixture in honeybee. Insect Mol. Biol. 8: 419-421.

Grabherr MG, Haas BJ, Yassour M, Levin JZ, et al. (2011). Full-length transcriptome assembly from RNA-Seq data without a reference genome. Nat. Biotechnol. 29: 644-652.

Groh C, Ahrens D and Rossler W (2006). Environment- and age-dependent plasticity of synaptic complexes in the mushroom bodies of honeybee queens. Brain Behav. Evol. 68: 1-14.

Guzman-Novoa E, Emsen B, Unger P, Espinosa-Montaño LG, et al. (2011). Genotypic variability and relationships between mite infestation levels, mite damage, grooming intensity, and removal of Varroa destructor mites in selected strains of worker honey bees (Apis mellifera L.). J. Invertebr. Pathol. 110: 314-320.

Martin C, Provost E, Roux M, Bruchou C, et al. (2001). Resistance of the honey bee, Apis mellifera to the acarian parasite Varroa destructor: behavioural and electroantennographic data. Physiol. Entomol. 26: 362-370.

McQuillan HJ, Nakagawa S and Mercer AR (2012). Mushroom bodies of the honeybee brain show cell population-specific plasticity in expression of amine-receptor genes. Learn. Mem. 19: 151-158.

Mortazavi A, Williams BA, McCue K, Schaeffer L, et al. (2008). Mapping and quantifying mammalian transcriptomes by RNA-Seq. Nat. Methods 5: 621-628.

Navajas M, Migeon A, Alaux C, Martin-Magniette M, et al. (2008). Differential gene expression of the honey bee Apis mellifera associated with Varroa destructor infection. BMC Genomics 9: 301.

O'Connell K, Gannon J, Doran P and Ohlendieck K (2008). Reduced expression of sarcalumenin and related $\mathrm{Ca}^{2+}$ -regulatory proteins in aged rat skeletal muscle. Exp. Gerontol. 43: 958-961.

Park JM, Kunieda T and Kubo T (2003). The activity of Mblk-1, a mushroom body-selective transcription factor from the honeybee, is modulated by the ras/MAPK pathway. J. Biol. Chem. 278: 18689-18694.

Parker R, Guarna MM, Melathopoulos AP, Moon KM, et al. (2012). Correlation of proteome-wide changes with social 
immunity behaviors provides insight into resistance to the parasitic mite, Varroa destructor, in the honey bee (Apis mellifera). Genome Biol. 13: R81.

Rath W and Drescher W (1990). Response of Apis cerana Fabr towards brood infested with Varroa jacobsoni Oud infestation rate of colonies in Thailand. Apidologie 21: 311-321.

Robinson GE (2004). Genomics. Beyond nature and nurture. Science 304: 397-399.

Rosenkranz P, Aumeier P and Ziegelmann B (2009). Biology and control of Varroa destructor. J. Invertebr. Pathol. 103 (Suppl 1): S96-S119.

Sørensen TL, Møller JV and Nissen P (2004). Phosphoryl transfer and calcium ion occlusion in the calcium pump. Science 304: $1672-1675$.

Swanson JA, Torto B, Kells SA, Mesce KA, et al. (2009). Odorants that induce hygienic behavior in honeybees: identification of volatile compounds in chalkbrood-infected honeybee larvae. J. Chem. Ecol. 35: 1108-1116.

Takeuchi H, Kage E, Sawata M, Kamikouchi A, et al. (2001). Identification of a novel gene, Mblk-1, that encodes a putative transcription factor expressed preferentially in the large-type Kenyon cells of the honeybee brain. Insect Mol. Biol. 10: 487-494.

Toyoshima $\mathrm{C}$ and Nomura $\mathrm{H}$ (2002). Structural changes in the calcium pump accompanying the dissociation of calcium. Nature 418: 605-611.

Tsuruda JM, Harris JW, Bourgeois L, Danka RG, et al. (2012). High-resolution linkage analyses to identify genes that influence Varroa sensitive hygiene behavior in honey bees. PLoS One 7: e48276.

Warburton EC, Glover CP, Massey PV, Wan H, et al. (2005). cAMP responsive element-binding protein phosphorylation is necessary for perirhinal long-term potentiation and recognition memory. J. Neurosci. 25: 6296-6303.

Warrit N and Lekprayoon C (2011). Honeybees of Asia. Springer Berlin Heidelberg, Berlin.

Yao B, Zhao Y, Zhang H, Liu M, et al. (2012). Sequencing and de novo analysis of the Chinese Sika deer antler-tip transcriptome during the ossification stage using Illumina RNA-Seq technology. Biotechnol. Lett. 34: 813-822.

Yoshida M, Minamisawa S, Shimura M, Komazaki S, et al. (2005). Impaired $\mathrm{Ca}^{2+}$ store functions in skeletal and cardiac muscle cells from sarcalumenin-deficient mice. J. Biol. Chem. 280: 3500-3506.

Zhao X, Yoshida M, Brotto L, Takeshima H, et al. (2005). Enhanced resistance to fatigue and altered calcium handling properties of sarcalumenin knockout mice. Physiol. Genomics 23: 72-78.

Zhou T (2005). The Biological Characteristics and the Natural Distribution of Varroa destructor in China. China Agricutural University, Beijing. 